\title{
Anatomic characteristics of supraorbital and supratrochlear nerves relevant to their use in corneal neurotization
}

\author{
Leahthan F. Domeshek ${ }^{1} \cdot$ Daniel A. Hunter ${ }^{1} \cdot$ Katherine Santosa $^{1} \cdot$ Steven M. Couch ${ }^{2} \cdot$ Asim Ali $^{3}$. \\ Gregory H. Borschel ${ }^{4} \cdot$ Ronald M. Zuker ${ }^{4} \cdot$ Alison K. Snyder-Warwick $^{1}$
}

Received: 24 June 2018 / Accepted: 27 August 2018 / Published online: 27 September 2018

(c) The Royal College of Ophthalmologists 2018

\begin{abstract}
Background Corneal denervation can lead to opacification and blindness. A new treatment technique, surgical corneal neurotization, transfers healthy donor nerve, (most commonly contralateral supratrochlear or supraorbital) to the affected limbus to prevent corneal destruction and improve healing potential of the cornea following insult. We examine gross and histomorphometric anatomy of the supratrochlear and supraorbital nerves relevant to their use in corneal neurotization.

Methods For each of nine adult cadaver heads, bilateral supraorbital and supratrochlear nerves were dissected from the supraorbital rim to the anterior hairline. The following data were recorded for each nerve: exit from the orbit through a notch versus foramen; horizontal distance from midline at the supraorbital rim; and distance from orbital exit to first branching point. Samples of all left supraorbital and supratrochlear nerves were obtained at the level of the supraorbital rim and at points $3 \mathrm{~cm}$ and $6 \mathrm{~cm}$ distally for histomorphometric analysis. Myelinated axon counts were determined for each sample. Results Four supraorbital foramina, 14 supraorbital notches, two supratrochlear foramina, and 15 supratrochlear notches were identified. Average supraorbital and supratrochlear distances to midline were $26.5 \mathrm{~mm}$ and $21 \mathrm{~mm}$ respectively. Average myelinated axon counts for both nerves were greater at the orbital rim (supraorbital: 6018, supratrochlear: 2533) than at $6 \mathrm{~cm}$ distally (supraorbital: 1621, supratrochlear: 1112).

Conclusions Anatomic dissection shows relative close approximation of the supraorbital and supratrochlear nerves, with a high proportion of both nerves exiting the orbit through foramina. The supraorbital nerve at the orbital rim contains the greatest number of myelinated axons.
\end{abstract}

Presented at: American Society for Peripheral Nerve (ASPN) 2016 annual meeting in Scottsdale, AZ.

Alison K. Snyder-Warwick

snydera@wustl.edu

1 Division of Plastic and Reconstructive Surgery, Washington University School of Medicine, St. Louis, MO, USA

2 Department of Ophthalmology, Washington University School of Medicine, St. Louis, MO, USA

3 Department of Ophthalmology and Vision Sciences, The Hospital for Sick Children, University of Toronto, Toronto, ON, Canada

4 Division of Plastic Surgery, The Hospital for Sick Children, University of Toronto, Toronto, ON, Canada

\section{Introduction}

The cornea, the primary refractive surface and mechanical barrier of the eye, is one of the most densely innervated tissues in the body [1,2]. Corneal sensation, supplied by the ophthalmic division of the trigeminal nerve, is responsible for maintaining a normal tear film and preserving healthy corneal epithelium via modulation of homeostasis and wound healing [2]. Damage to this epithelium, the eye's most superficial barrier against external insults and an integral component of the cornea-tear-film interface, can lead to ulceration and opacify this delicate transparent structure which threatens vision.

Neurotrophic keratopathy (NK, Fig. 1) is a potentially devastating disorder caused by congenital or acquired dysfunction of the ophthalmic division of the trigeminal nerve. With NK, lack of sensory innervation predisposes the corneal epithelium to breakdown and impairs epithelial healing following injury [3, 4], leading to persistent corneal 


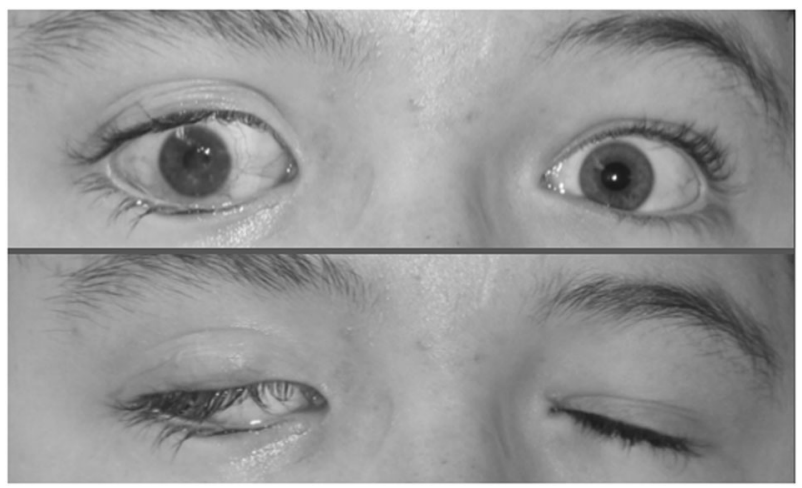

Fig. 1 Neurotrophic keratopathy (top panel) resulting from dysfunction of the ophthalmic division of the trigeminal nerve can lead to corneal degradation ranging in severity from mild epithelial irregularities to ulceration, perforation, and even blindness. Facial nerve palsy, which can result in lagophthalmos (bottom panel) is often seen in conjunction with $\mathrm{NK}$, placing the cornea at greater risk for damage given the increased exposure in the setting of corneal anesthesia

ulceration, corneal opacification, and blindness. Conservative treatment for NK remains challenging, and surgical intervention aims to protect ocular integrity rather than improve or preserve vision [4]. Furthermore, once damage leads to irreversible scarring and loss of vision, patients with NK are not ideal candidates for corneal transplantation because without sensory innervation, a transplanted cornea would be subject to the same fate, requiring multiple transplants.

Surgical neurotization is a revolutionary treatment that directly addresses the etiology of NK to offer a potential cure. When successful, it facilitates corneal healing and prevention of corneal breakdown, while preserving vision and decreasing reliance on medications [5-7]. Additionally, it can transform patients with severe, irreversible corneal scarring into better corneal transplant candidates. Multiple techniques for corneal reinnervation have been described utilizing branches of the occipital or trigeminal nerves. The first description of corneal neurotization with the trigeminal nerve involved direct transfer of the distal branches of the contralateral supraorbital (SO) or supratrochlear (ST) nerves to the limbus of the affected eye [5, 8]. More recently, corneal neurotization utilizing a sural nerve graft interposed between the proximal donor SO or ST nerve and the affected limbus has been described (Fig. 2) [6, 7]. Restoration of corneal sensation has been reported following neurotization [5-8]. Patient outcomes have been very encouraging, but reported data include variability in time to sensory recovery and degree of ultimate sensation, as well as differences in patient populations and etiologies of sensory loss between those treated with direct and graft neurotization.

Corneal neurotization is a surgical technique still in its infancy. As methods evolve and diversify, understanding

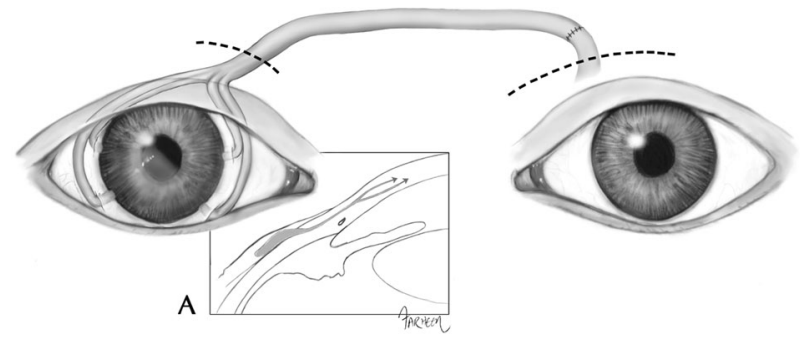

Fig. 2 Surgical corneal neurotization with the use of an interposed nerve graft from the donor supraorbital or supratrochlear nerve (left eye in the figure) to the limbus of the affected eye (right eye in the figure). The dashed lines above the two eyes indicate the upper eyelid incisions through which the donor nerve (left eye) is harvested and through which the limbus is accessed (right eye). This depiction shows an end-to-end coaptation between donor nerve and nerve graft, although it is also possible to coapt the end of the nerve graft to the side of the donor nerve. Box A shows the right eye in cross-section, with the nerve graft (yellow) tunneled into the limbus and growing (indicated by red arrows) into the cornea

relevant anatomy will facilitate surgical planning in order to optimize functional outcomes. Many factors affect the success of nerve transfers, including donor nerve axon count, the use of nerve grafts, and the distance from donor to reinnervation target. Herein, we examine SO and ST gross and histomorphometric neuroanatomy relevant to their use in corneal neurotization.

\section{Materials and methods}

\section{Specimens and dissection}

After obtaining Institutional Review Board exemption, dissections were performed on nine adult cadaver heads. For each specimen, bilateral SO and ST nerves were accessed through a bicoronal approach, with subperiosteal dissection to the supraorbital rim. Under loupe magnification, each SO and ST nerve was identified and dissected free from surrounding tissues in order to visualize the exit of the nerve at the supraorbital rim. For each specimen, the following data were recorded for all four nerves: the presence of notch versus foramen at the supraorbital rim; horizontal distance from midline at the supraorbital rim; and distance from the exit of the nerve to its first branching point.

\section{Histomorphometric analysis}

Histomorphometric analyses were performed at three points along each left SO nerve and left ST nerve to determine axon counts. Histomorphometry was assessed from nerve samples located: (1) at the supraorbital rim, (2) $3 \mathrm{~cm}$ distal (superior) to the rim, and (3) $6 \mathrm{~cm}$ distal to the rim, which approximated the anterior hairline in most specimens 


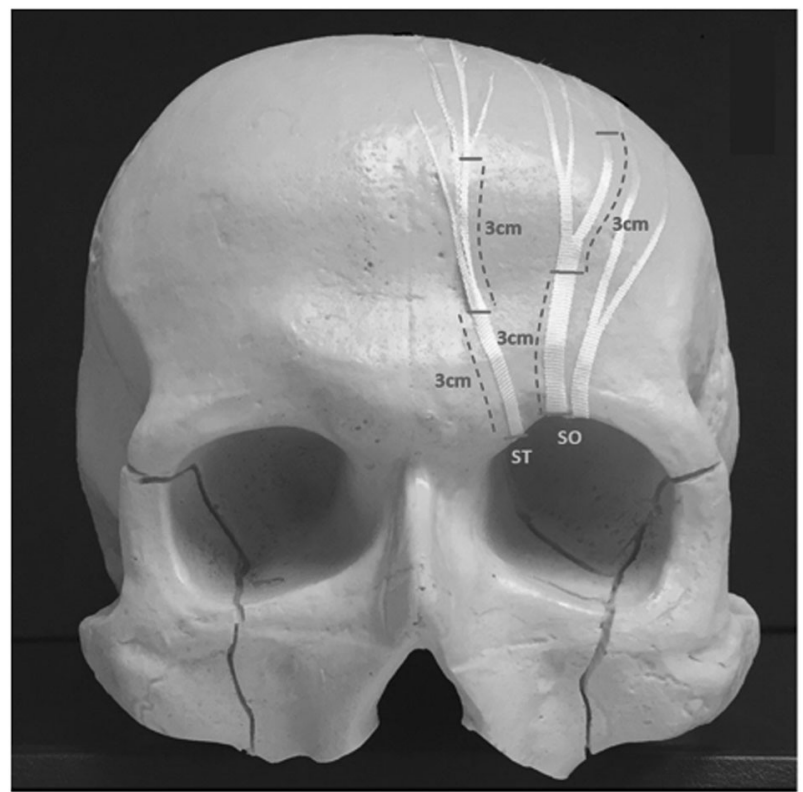

Fig. 3 Supraorbital and supratrochlear nerve harvest sites. Samples of left supraorbital and supratrochlear nerves were harvested for histologic analysis at three locations each (horizontal red hashmarks): their exit from the superior orbital rim, $3 \mathrm{~cm}$ distal to the rim, and $6 \mathrm{~cm}$ distal to the rim. Only one branch of each nerve (usually the largest identifiable) was sampled at each location

(Fig. 3). When nerves were found to exit the rim as multiple branches, only one branch (the branch appearing grossly to be the largest) was harvested for axon counts. Similarly, when multiple nerve branches were noted distally, the branch that grossly appeared to be the largest was selected for histomorphometric analysis, as multiple small-caliber nerve branches had divergent courses that would likely preclude clinical use as donor nerves and complicate histomorphometric analyses.

Nerve specimens were fixed in a cold, buffered 3\% glutaraldehyde solution for $24 \mathrm{~h}$, post-fixed with osmium tetroxide, and embedded in Araldite 502 (Polysciences Inc., Warrington, PA). One micrometre-thick cross-sections were cut with an LKB III Ultramicrotome (LKB-Produkter A.B., Bromma, Sweden) and stained with $1 \%$ toluidine blue. Under light microscopy, stained cross-sections were evaluated for overall nerve architecture.

Using an automated digital image-analysis system linked to morphometry macros developed for peripheral nerve analysis (Leco Instruments, St. Joseph, Michigan), the microscope image was digitized and displayed on a video monitor with a calibration of $0.125 \mu \mathrm{m} /$ pixel. Binary histomorphometry analyses of the digitized information based on gray and white scales allowed measurements of total fascicular area and total fiber number in the nerves. At 1000x magnification, 7-13 randomly selected fields per nerve, or a minimum of 500 myelinated fibers, were counted. From these, calculations of nerve fiber density (fibers $/ \mathrm{mm}^{2}$ ) and total number of myelinated fibers in the neural tissue were performed. A scientist blinded to the experimental groups performed all measurements.

\section{Statistical analysis}

T-tests were performed on axon counts to evaluate differences in counts at the orbital rim and $6 \mathrm{~cm}$ distally and to evaluate differences between SO and ST counts at the orbital rim. $P$-values $<0.05$ were defined as significant.

\section{Results}

\section{Gross anatomic characteristics}

SO and ST nerves were identified for all except two specimens - one of which had no identifiable ST nerve bilaterally, and one of which had no identifiable ST nerve on the right only. In one specimen in which both SO and ST nerves were identified bilaterally, both nerves exited through one large notch bilaterally. In all other specimens both SO and ST nerves were identified, and the nerves had distinct exits. Fourteen (78\%) of the 18 SO nerves and 15 $(88 \%)$ of the 17 ST nerves exited the orbits through notches, with the remaining exiting through foramina. Bony anatomy was not necessarily symmetric for a given cadaver (Fig. 4). Average distance from nerve to midline at the level of the supraorbital rim was $26.5 \pm 2.7 \mathrm{~mm}$ and $21.0 \pm 4.0 \mathrm{~mm}$ for SO and ST nerves respectively.

Average distance from supraorbital rim to the first branch was $2.4 \pm 3.5 \mathrm{~mm}$ and $7.2 \pm 3.1 \mathrm{~mm}$ for SO and ST nerves respectively. Eleven of the 18 dissected SO nerves branched immediately upon exit from the supraorbital rim (at $0 \mathrm{~mm}$ ). Of the SO nerves that branched immediately on exit from the rim, 8 exited as 2 branches, and 2 exited as 3 branches. None of the ST nerves branched immediately upon exit from the supraorbital rim, and 3 of the 15 identified ST nerves had no identifiable branches between the supraorbital rim and the anterior hairline.

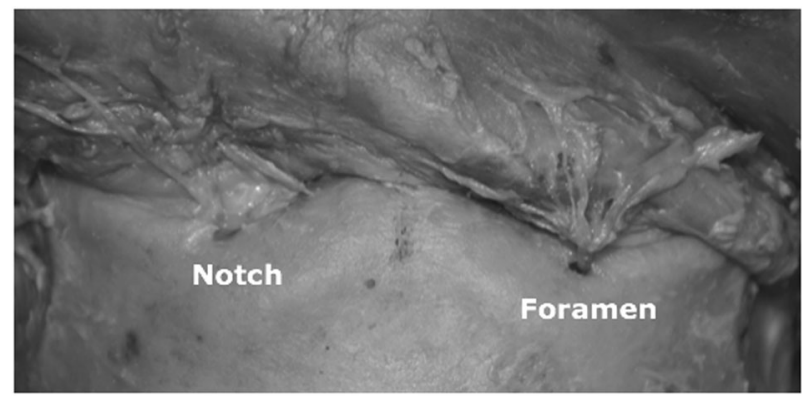

Fig. 4 Most supraorbital (SO) and supratrochlear (ST) nerves exit the supraorbital rim through notches. Bony anatomy at the supraorbital rim is not necessarily symmetric for a given patient 


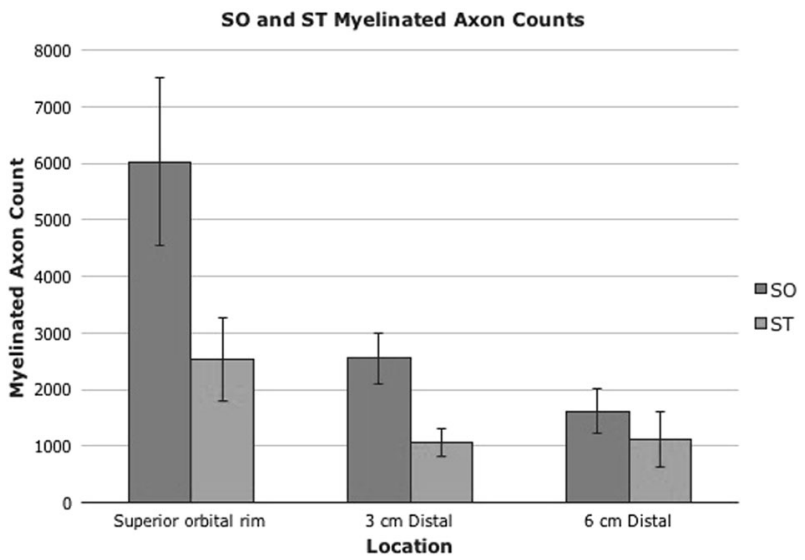

Fig. 5 For both supraorbital (SO) and supratrochlear (ST) nerves, average myelinated axon counts were greatest proximally. The most precipitous drop in axons occurred proximally, with a nearly $60 \%$ decrease in $\mathrm{SO}$ axon count by $3 \mathrm{~cm}$ distally. By $6 \mathrm{~cm}$, average $\mathrm{SO}$ axon count was less than one third of that at the supraorbital rim. Throughout their courses, the SO nerves contained a greater number of axons than did ST nerves. The difference was largest proximally, with the SO nerve containing on average greater than double the number of axons of the ST nerve at the supraorbital rim

\section{Histomorphologic analyses}

Average myelinated axon counts for SO and ST samples at the rim, $3 \mathrm{~cm}$, and $6 \mathrm{~cm}$ distally are depicted in Fig. 5 . At the supraorbital rim, SO and ST nerves contained on average $6017 \pm 4195$ (standard deviation) fibers and $2534 \pm 1667$ myelinated axons respectively. Both nerves contained more axons proximally, as would be expected given branching of the nerves as they travel distally. The most precipitous drop in axons occurred proximally, with a nearly $60 \%$ decrease in SO axon count in the initial $3 \mathrm{~cm}$ from the orbital rim. By $6 \mathrm{~cm}$ distally, average $\mathrm{SO}$ axon count was less than one third of that at the supraorbital rim (1620 \pm 1046 myelinated axons). Data were similar for the SO and ST nerves. The difference in axon counts at the rim and $6 \mathrm{~cm}$ distally was statistically significant $(p<0.05)$ for SO but not ST nerves $(p=0.15)$. Throughout their courses, the SO nerves contained a greater number of axons than did ST nerves. The difference was largest proximally, with the SO nerve containing on average greater than double the number of axons as the ST nerve at the supraorbital rim. The difference between SO and ST axon counts at the rim was not statistically significant $(p=0.06)$, but the statistics may be affected by the small number of specimens included in this study.

\section{Discussion}

The SO nerve at the orbital rim was found to be the most robust axonal source, containing greater than double the number of ST fibers at the same location, and more than triple the number of SO or ST fibers $6 \mathrm{~cm}$ distally. Even with the use of an interposed nerve graft, based on these findings, the proximal SO nerve can be expected to remain a favorable donor for corneal neurotization.

Clinically, both SO and ST nerves have been used to restore corneal sensation, through both direct transfer and nerve graft neurotization [5-7]. Each surgical technique offers unique advantages and disadvantages. Direct neurotization involves an extensive bicoronal incision, which is a source of morbidity and potential cosmetic concern, but spares the patient a second surgical site and the donor morbidity associated with nerve graft harvest. Graft neurotization, performed through well-hidden upper eyelid incisions, obviates the need for a bicoronal incision, but at the cost of an additional, albeit generally well-tolerated [9], nerve graft donor site. Use of a nerve graft also offers versatility and may be used to restore sensation in cases of bilateral corneal anesthesia [6,7], especially in cases in which only one donor forehead dermatome is sensate.

There is a density-dependent effect of axons on corneal function, with dry eye symptoms and impaired sensation related to decreased corneal nerve density and altered anatomy of the subbasal plexus [10]. Exact axonal thresholds for protective or normal sensation are unknown, but studies of postoperative recovery following refractive procedures such as keratoplasty and laser-assisted in situ keratomileusis (LASIK) provide insight into the relationship between nerve supply and corneal function. Incisional refractive keratoplasty disrupts the subbasal plexus, and patients experience postoperative dry eye symptoms and decreased corneal sensitivity. The plexus recovers slowly, and studies suggest incomplete recovery even 3 years following surgery [11]. Despite persistent damage, however, normal corneal sensation generally returns within 6-12 months postoperatively, even with well below $50 \%$ recovery of the subbasal plexus [11, 12].

Corneal neurotization is capable of transferring sufficient donor axons to generate functional results [5-7]. However, no technique has proven ubiquitously effective and reasons for variability in results have not been fully elucidated. Many patient and technical factors (in addition to axonal threshold for target function) influence the success of nerve transfers, including duration and etiology of target denervation, patient age and health, distance to reinnervation, use of a nerve graft, and donor nerve axonal load [13]. Though an anatomic study alone cannot define optimal technique, it enhances understanding of some of the above factors to facilitate prediction of axonal transmission and therefore relative likelihood of functional reinnervation based on procedure and patient circumstance.

Although our analyses indicate a steep drop off in axon count with increasing distance from the orbital rim, the 
presented numbers indicate only counts for a single branch of each nerve at each distance. Were axons from all branches included, total axon counts on exit from the orbit, as well as the extent of distal drop off likely would have differed. Our data do, however, demonstrate the trend of axon drop off relative to the main nerve branch with distance from the orbital rim. Axon counts at the orbital rim were based on only one nerve branch when multiple branches were noted upon exit. This method did not affect ST counts. However, many SO nerves emerged as multiple branches, such that for a given SO nerve, total axon count at the rim may have been double or triple that which we recorded, depending on branching pattern, and the difference between total SO and ST axons at the rim would be larger than those that we described. Distally, axon counts for both nerves were underestimated by our technique. It stands to reason that, since only one of many possible branches was sampled, true drop off in total axon count for both nerves would be less than that reported.

Despite the discrepancies between our counts and true numbers of SO and ST axons distally, for the purposes of corneal neurotization, the counts from individual branches likely remain the most clinically relevant. Coaptation between donor and graft in the graft technique is performed proximally, and most often involves only one donor nerve branch. In the event that the SO, rather than ST nerve, is utilized, the multiple SO branches at the level of coaptation are likely too spread out for use of more than one in an endto-side coaptation. If an end-to-end coaptation is utilized, then use of greater than one branch will result in greater frontal anesthesia, and potentially a size mismatch between donor and graft. In direct neurotization, multiple distal ST and SO branches may be harvested, though the number used may differ from case to case, and the number of donor axons is easily estimated by totaling expected axon counts for each branch transferred.

Previous studies have examined SO and ST nerve morphology at the orbital rim, frequently looking towards the relevance of this anatomy to cosmetic and migraine procedures [14-18]. These reports do not address axonal loads, a key consideration for corneal neurotization, but their descriptions of gross anatomy provide points of comparison for, and adjuncts to, our gross anatomic findings.

In corneal neurotization, the bony exits of these nerves can affect donor nerve mobility, and therefore length of nerve needed for tension-free transfer to the contralateral limbus. Both nerves have been noted to emerge most often through notches, with a stronger predominance reported for ST (72-100\% through notches) than for SO nerves (50-83.3\% through notches) $[17,19]$, similar to our findings (93 and $78 \%$ notches for ST and SO nerves, respectively). Foramina are potential points of nerve compression $[15,17]$ that may lead to ischemic changes and subsequent demyelination and even axonal degeneration [20, 21]. Preventable sources of axonal damage, including pressure and kinking, must be avoided. The presence of a foramen rather than a notch does not preclude utilization of a particular donor nerve for corneal neurotization, however if a foramen is constrictive around the nerve or restrictive to transfer (i.e. kinking occurs with redraping of the nerve in a direct transfer), osteotomies or more extensive donor nerve mobilization should be considered to optimize axonal delivery.

ST and SO distances from midline at the rim are consistent throughout the literature. Though one group found the ST nerve to emerge an average of $8.7 \mathrm{~mm}$ lateral to midline [19], other authors report averages of 17.5-19.7 mm $[15,18]$, similar to our measurement of $21.0 \mathrm{~mm}$. Our average SO nerve measurement ( $26.5 \mathrm{~mm}$ from midline) also agrees with the previously reported range of $19.2 \mathrm{~mm}$ to $26.1 \mathrm{~mm}[18,19]$. We did find variability in both absolute distances from midline at which the two nerves emerge as well as the gap between the exits of the two nerves. In one specimen, both the SO and ST nerves emerged from one large notch bilaterally, a phenomenon noted by others to occur in $6 \%$ of orbits [15].

What then are the implications of projected graft length for likelihood of success of graft neurotization utilizing SO versus ST nerves? Regarding selection of donor nerve for graft neurotization: use of the SO nerve may provide better clinical outcomes. The greater robustness of the proximal SO as opposed to ST nerve should more than offset additional axonal drop off that may occur along extra graft length required for transfer of SO as opposed to ST nerves. Given the SO nerve's early branching pattern and steep decline in axon count shortly after exit from the orbit, dissection of the nerve to the orbital rim and coaptation as proximally as possible will maximize axonal supply.

This study provides a starting point for discussing the anatomic basis of surgical planning in corneal neurotization. Over recent decades, there has been an exponential increase in our understanding of nerve regeneration in general and the application of nerve grafting and transfers throughout the body. In contrast, neurotization of the cornea specifically is a relatively new endeavor, and many gaps exist in our understanding of the mechanisms underlying clinical results. We have much to learn about the neurophysiology of these transfers - the ingrowth of transferred nerves into the affected cornea, effects of duration and etiology of corneal injury on functional results, and the fate of unmyelinated donor axons in corneal neurotization. As understanding of these factors improves, so will techniques for corneal neurotization that will allow surgeons to optimize outcomes and minimize morbidity. 


\section{Summary}

\section{What was known before}

- Both supraorbital and supratrochlear nerves have been used successfully to provide sensation through corneal neurotization

- Larger nerves contain a greater number of axons to donate through transfers to power (or provide sensation to) their targets

\section{What this study adds}

- Axon counts and relative sizes of the supraorbital and supratrochlear nerves for enhanced understanding of donor nerve potential

- Description of gross anatomic aspects (i.e. distance from midline of nerve exits from the orbital rim and distance to first nerve branch) relevant to corneal neurotization to assist with surgical planning

Acknowledgements We would like to thank Farheen Ali for her illustration of corneal neurotization.

\section{Compliance with ethical standards}

Conflict of interest The authors declare that they have no conflict of interest

\section{References}

1. Muller LJ, Marfurt CF, Kruse F, Tervo TM. Corneal nerves: structure, contents and function. Exp Eye Res. 2003;76:521-42.

2. Kowtharapu BS, Stahnke T, Wree A, Guthoff RF, Stachs O. Corneal epithelial and neuronal interactions: role in wound healing. Exp Eye Res. 2014;125:53-61.

3. Hsu HY, Modi D. Etiologies, quantitative hypoesthesia, and clinical outcomes of neurotrophic keratopathy. Eye Contact Lens. 2015;41:314-7.

4. Sacchetti M, Lambiase A. Diagnosis and management of neurotrophic keratitis. Clin Ophthalmol. 2014;8:571-9.
5. Terzis JK, Dryer MM, Bodner BI. Corneal neurotization: a novel solution to neurotrophic keratopathy. Plast Reconstr Surg. 2009;123:112-20.

6. Elbaz U, Bains R, Zuker RM, Borschel GH, Ali A. Restoration of corneal sensation with regional nerve transfers and nerve grafts: a new approach to a difficult problem. JAMA Ophthalmol. 2014; 132:1289-95.

7. Bains RD, Elbaz U, Zuker RM, Ali A, Borschel GH. Corneal neurotization from the supratrochlear nerve with sural nerve grafts: a minimally invasive approach. Plast Reconstr Surg. 2015; $135: 397 \mathrm{e}-400 \mathrm{e}$.

8. Samii M. Reconstruction of the trigeminal nerve. In: Samii M, Janetta PJ, ed. The cranial nerves. New York: Springer-Verlag Berlin Heidelberg; 1981.

9. Hallgren A, Bjorkman A, Chemnitz A, Dahlin LB. Subjective outcome related to donor site morbidity after sural nerve graft harvesting: a survey in 41 patients. BMC Surg. 2013;13:39.

10. Kheirkhah A, Dohlman TH, Amparo F, et al. Effects of corneal nerve density on the response to treatment in dry eye disease. Ophthalmology . 2015;122:662-8.

11. Calvillo MP, McLaren JW, Hodge DO, Bourne WM. Corneal reinnervation after LASIK: prospective 3-year longitudinal study. Invest Ophthalmol Vis Sci. 2004;45:3991-6.

12. Lee BH, McLaren JW, Erie JC, Hodge DO, Bourne WM. Reinnervation in the cornea after LASIK. Invest Ophthalmol Vis Sci. $2002 ; 43: 3660-4$.

13. Tung TH, Mackinnon SE. Nerve transfers: indications, techniques, and outcomes. J Hand Surg Am. 2010;35:332-41.

14. Knize DM. A study of the supraorbital nerve. Plast Reconstr Surg. 1995;96:564-9.

15. Janis JE, Hatef DA, Hagan R, et al. Anatomy of the supratrochlear nerve: implications for the surgical treatment of migraine headaches. Plast Reconstr Surg. 2013;131:743-50.

16. Janis JE, Ghavami A, Lemmon JA, Leedy JE, Guyuron B. The anatomy of the corrugator supercilii muscle: part II. Supraorbital nerve branching patterns. Plast Reconstr Surg. 2008;121:233-40.

17. Fallucco M, Janis JE, Hagan RR. The anatomical morphology of the supraorbital notch: clinical relevance to the surgical treatment of migraine headaches. Plast Reconstr Surg. 2012;130:1227-33.

18. Miller TA, Rudkin G, Honig M, Elahi M, Adams J. Lateral subcutaneous brow lift and interbrow muscle resection: clinical experience and anatomic studies. Plast Reconstr Surg. 2000; 105:1120-7. discussion 1128

19. Konofaos P, Soto-Miranda MA, Ver Halen J, Fleming JC. Supratrochlear and supraorbital nerves: an anatomical study and applications in the head and neck area. Ophthalmic Plast Reconstr Surg. 2013;29:403-8.

20. Mackinnon SE, Dellon AL, Hudson AR, Hunter DA. Chronic human nerve compression--a histological assessment. Neuropathol Appl Neurobiol. 1986;12:547-65.

21. Rempel DM, Diao E. Entrapment neuropathies: pathophysiology and pathogenesis. J Electromyogr Kinesiol. 2004;14:71-75. 